\title{
High spatial resolution XPS and AES applications in understanding microscopic surface phenomena
}

\author{
H. Piao and L. Le Tarte
}

General Electric Co. Global Research Center, Niskayuna, NY, 12309

X-ray Photoelectron Spectroscopy (XPS) and Auger Electron Spectroscopy (AES) are the most widely used techniques for quantitative surface analysis. The XPS is mainly dedicated to the identification of chemical composition/bonding on surfaces, while the high spatial resolution in AES has made it an established technique for determining the localized defects or other features distributed on surfaces. Although the spatial resolution in XPS has been much inferior to that in AES, the recent development of XPS instrumentation with near-micron spatial resolution has advanced the capability of elemental and chemical state imaging accompanied by small area analysis [1]. The goal of this presentation is to present how the combined uses of XPS and AES at enhanced spatial resolution have significantly improved the understanding of the chemistry of heterogeneous surfaces, particularly from the fields such as microelectronic materials/devices, thin films and coatings.

Figure 1 shows an optical image of a part of a microelectronic device. The photo exhibited the occurrence of delamination on some pads. Examination of the delaminated surface seems to be limited to the utilization of AES in consideration of the small localized features on the surface. In fact, Auger analysis (with the electron beam size less than 50 $\mathrm{nm}$ ) clearly showed the ITO delamination (see Table 1). The detection of the nitrogen on the delaminated surface (area B) appears to indicate the polymer residuals left at the ITO/Mo interface during the fabrication. Figure 2 shows the quantitative XPS images of all the key elements. The localization of In and Mo are clearly revealed. In addition, the $\mathrm{C}$ and $\mathrm{N}$ are more enriched on the delaminated Mo surface. High resolution $\mathrm{C} 1 \mathrm{~s}$ and N1s studies (chemical bonding information, not shown here) at selected small areas $(27 \mu \mathrm{m})$ further support the presence of polymer residual at the ITO/Mo interface, which is most likely responsible for the ITO delamination. As can be clearly seen, the XPS at improved spatial resolution is well suited for these investigations.

In the second part of the presentation, the mathematical procedure using Principle Component Analysis (PCA) in the reduction of noise in XPS images [2] will be described. The purpose of the PCA is to identify the abstract factor images containing significant information and reconstruct the images only using these factors without noise, therefore, resulting in the set of images with enhanced signal-to-noise. The dramatic improvements in the image contrast and chemical component determination will be demonstrated.

\section{References}

[1] D. Briggs and J.T. Grant, Surface Analysis by Auger and X-ray Photoelectron Spectroscopy, Surface Spectra/IMPublications, 2003.

[2] N. Fairley and A. Carrick, The Casa Cookbook, Acolyte Science, 2005. 


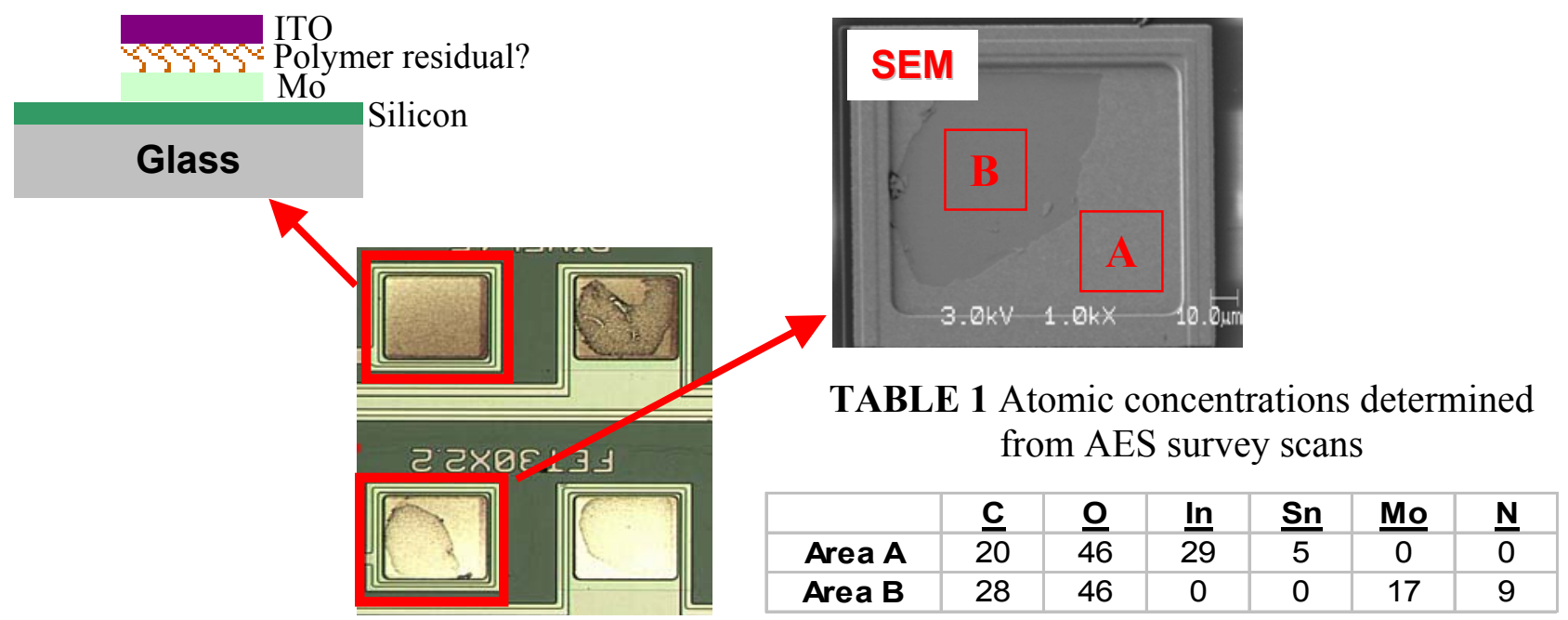

FIG. 1. The optical image (center) and the SEM image (right) of a microelectronic device that showing the delamination occurred at some pad areas. A schematic of the pad structure is also shown on the left.
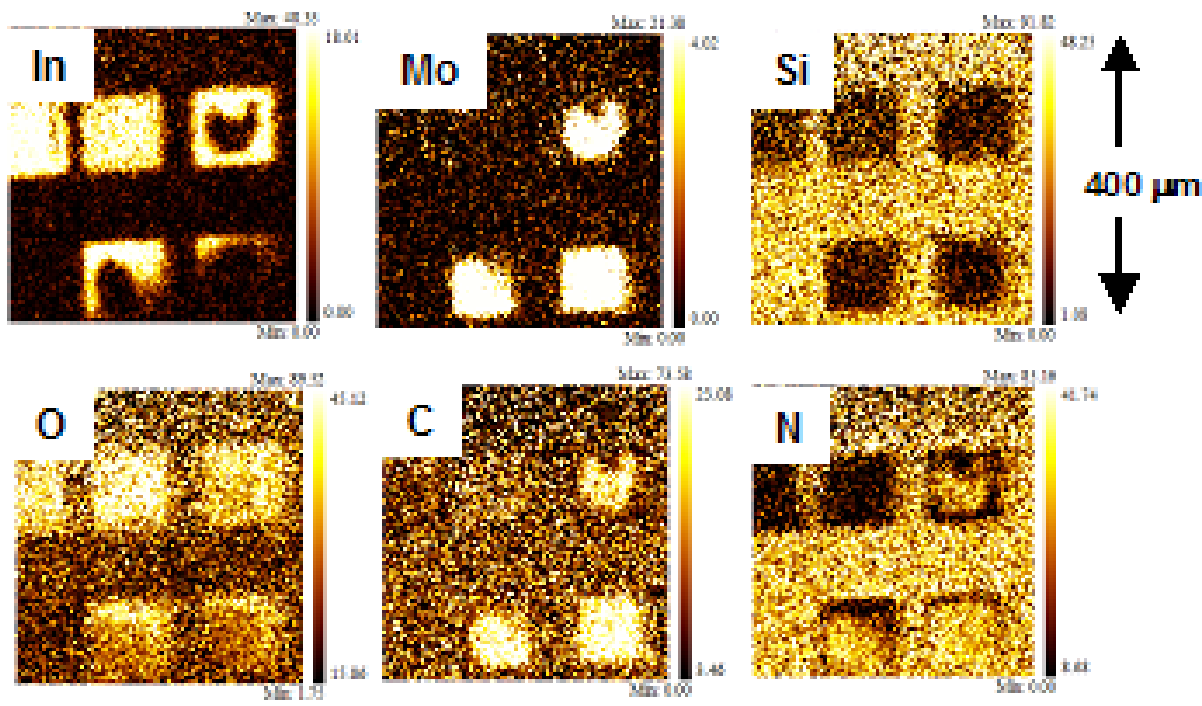

FIG. 2. Quantified XPS images of the key elements (In, Mo, Si, O, C and N) present on the device surface. The images were sharpened through the Principle Component Analysis (PCA) of the spectral image data set. 